\title{
On the extension of Hilbert Inequality for Finite Series \\ Baoju Sun
}

Department of Mathematics

Zhejiang Water Conservancy \& Hydropower University, Hangzhou, Zhejiang 310018, China sunbj@zjweu.edu.cn

Keywords: Hilbert inequality; Cauchy inequality; Hadamard inequality

Abstract. In this paper, by introducing parameter $\lambda$, and using Hadamard inequality and Cauchy inequality, an extension of Hilbert inequality with finite version is established.

\section{Introduction}

If $a_{n} \geq 0, b_{n} \geq 0$, such that $0<\sum_{n=1}^{\infty} a_{n}^{2}<\infty, 0<\sum_{n=1}^{\infty} b_{n}^{2}<\infty$, then the well known Hilbert inequality and its equivalent inequalities are given by

$$
\begin{gathered}
\sum_{n=1}^{\infty} \sum_{m=1}^{\infty} \frac{a_{m} b_{n}}{m+n}<\pi\left(\sum_{n=1}^{\infty} a_{n}^{2}\right)^{1 / 2}\left(\sum_{n=1}^{\infty} b_{n}^{2}\right)^{1 / 2} . \\
\sum_{n=1}^{N} \sum_{m=1}^{N} \frac{a_{m} b_{n}}{m+n}<\pi\left(\sum_{n=1}^{N} a_{n}^{2}\right)^{1 / 2}\left(\sum_{n=1}^{N} b_{n}^{2}\right)^{1 / 2} . \\
\sum_{n=0}^{\infty} \sum_{m=0}^{\infty} \frac{a_{m} b_{n}}{m+n+1}<\pi\left(\sum_{n=0}^{\infty} a_{n}^{2}\right)^{1 / 2}\left(\sum_{n=0}^{\infty} b_{n}^{2}\right)^{1 / 2} . \\
\sum_{n=0}^{N} \sum_{m=0}^{N} \frac{a_{m} b_{n}}{m+n+1}<\pi\left(\sum_{n=0}^{N} a_{n}^{2}\right)^{1 / 2}\left(\sum_{n=0}^{N} b_{n}^{2}\right)^{1 / 2} .
\end{gathered}
$$

(see Hardy et al.[1]). In recently years, various improvements and extensions of the Hilbert inequality and Hilbert type inequalities appear in a great deal of papers (see [2-5]). Zhang xiaoming, Chu yuming ([2]) gave improvements of (2),(4)as:

$$
\pi^{2} \sum_{n=1}^{N} a_{n}^{2} \sum_{n=1}^{N} b_{n}^{2}-\left(\sum_{n=1}^{N} \sum_{m=1}^{N} \frac{a_{m} b_{n}}{m+n}\right)^{2} \geq \min _{1 \leq n \leq N}\left\{n a_{n}^{2}\right\} \min _{1 \leq n \leq N}\left\{n b_{n}^{2}\right\}\left[\pi^{2}\left(\sum_{n=1}^{N} \frac{1}{n}\right)^{2}-\left(\sum_{n=1}^{N} \sum_{m=1}^{N} \frac{1}{\sqrt{m n}(m+n)}\right)^{2}\right]
$$

and

$$
\begin{aligned}
& \pi^{2} \sum_{n=0}^{N} a_{n}^{2} \sum_{n=0}^{N} b_{n}^{2}-\left(\sum_{n=0}^{N} \sum_{m=0}^{N} \frac{a_{m} b_{n}}{m+n+1}\right)^{2} \\
& \quad \geq \min _{0 \leq n \leq N}\left\{(n+1 / 2) a_{n}^{2}\right\} \min _{0 \leq n \leq N}\left\{(n+1 / 2) b_{n}^{2}\right\}\left[\pi^{2}\left(\sum_{n=0}^{N} \frac{1}{n+1 / 2}\right)^{2}-\left(\sum_{n=0}^{N} \sum_{m=0}^{N} \frac{1}{\sqrt{(m+1 / 2)(n+1 / 2)}(m+n)}\right)^{2}\right]
\end{aligned}
$$
(6).

\section{Some lemmas}

In order to prove our main result we need some lemmas, which we present in this section.

Lemma 1 (Hadamard inequality) $)^{[2]}$ Let $\varphi:[c, d] \rightarrow^{\circ}$ be a convex function, then one has

$$
\varphi\left(\frac{c+d}{2}\right) \leq \frac{1}{d-c} \int_{c}^{d} \varphi(x) d x \leq \frac{\varphi(c)+\varphi(d)}{2} .
$$


Lemma 2 If $1 \leq n \leq N, 0<\lambda \leq 2$, then

$$
\sum_{m=1}^{N} \frac{1}{m^{1-\lambda / 2}\left(m^{\lambda}+n^{\lambda}\right)}<\frac{\pi}{\lambda} n^{-\lambda / 2}
$$

Proof. Since $\frac{1}{x^{1-\lambda / 2}\left(x^{\lambda}+n^{\lambda}\right)}$ is monotone decreasing in the interval $0<x<\infty$, then

$$
\sum_{m=1}^{N} \frac{1}{m^{1-\lambda / 2}\left(m^{\lambda}+n^{\lambda}\right)}<\int_{0}^{N} \frac{1}{x^{1-\lambda / 2}\left(x^{\lambda}+n^{\lambda}\right)} \mathrm{d} x=\frac{1}{\lambda} n^{-\frac{\lambda}{2}} \int_{0}^{\left(\frac{N}{n}\right)^{\lambda}} \frac{1}{\sqrt{t}(1+t)} \mathrm{d} t<\frac{1}{\lambda} n^{-\frac{\lambda}{2}} \int_{0}^{\infty} \frac{1}{\sqrt{t}(1+t)} \mathrm{d} t=\frac{\pi}{\lambda} n^{-\frac{\lambda}{2}}
$$

Lemma 3 If $1 \leq n \leq N, 0<\lambda \leq 1$, then

$$
\sum_{m=0}^{N} \frac{1}{(m+1 / 2)^{1-\lambda / 2}\left((m+1 / 2)^{\lambda}+(n+1 / 2)^{\lambda}\right)}<\frac{\pi}{\lambda}(n+1 / 2)^{-\lambda / 2}
$$

Proof. Since $0<\lambda \leq 1$, The second derivative of the function

$$
\frac{1}{(x+1 / 2)^{1-\lambda / 2}\left((x+1 / 2)^{\lambda}+(n+1 / 2)^{\lambda}\right)} \text { is positive, then the function is a convex function, by using }
$$

Hadamard inequality, we have

$$
\begin{aligned}
& \frac{1}{(m+1 / 2)^{1-\lambda / 2}\left((m+1 / 2)^{\lambda}+(n+1 / 2)^{\lambda}\right)} \leq \int_{m-\frac{1}{2}}^{m+\frac{1}{2}} \frac{1}{(x+1 / 2)^{1-\lambda / 2}\left((x+1 / 2)^{\lambda}+(n+1 / 2)^{\lambda}\right)} d x, \\
& \sum_{m=0}^{N} \frac{1}{(m+1 / 2)^{-\lambda / 2}\left((m+1 / 2)^{\lambda}+(n+1 / 2)^{\lambda}\right)} \leq \sum_{m=0}^{N} \int_{m-\frac{1}{2}}^{m+\frac{1}{2}} \frac{1}{(x+1 / 2)^{1-\lambda / 2}\left((x+1 / 2)^{\lambda}+(n+1 / 2)^{\lambda}\right)} d x \\
& =\int_{-\frac{1}{2}}^{N+\frac{1}{2}} \frac{1}{(x+1 / 2)^{1-\lambda / 2}\left((x+1 / 2)^{\lambda}+(n+1 / 2)^{\lambda}\right)} d x=\frac{1}{\lambda}(n+1 / 2)^{-\frac{\lambda}{2}} \int_{0}^{\left(\frac{N+1}{n+1 / 2}\right)^{\lambda}} \frac{1}{\sqrt{t}(1+t)} d t \\
& <\frac{1}{\lambda}(n+1 / 2)^{-\frac{\lambda}{2}} \int_{0}^{\infty} \frac{1}{\sqrt{t}(1+t)} d t=\frac{\pi}{\lambda}(n+1 / 2)^{-\frac{\lambda}{2}} .
\end{aligned}
$$

This concludes the proof.

\section{Main results}

Theorem 1 If $a_{n} \geq 0, b_{n} \geq 0, n=1,2, \mathrm{~L}, N . \quad 0<\lambda \leq 2$, then

$$
\begin{aligned}
& \left(\frac{\pi}{\lambda}\right)^{2} \sum_{n=1}^{N} n^{1-\lambda} a_{n}^{2} \sum_{n=1}^{N} n^{1-\lambda} b_{n}^{2}-\left(\sum_{n=1}^{N} \sum_{m=1}^{N} \frac{a_{m} b_{n}}{m^{\lambda}+n^{\lambda}}\right)^{2} \\
& \geq \min _{1 \leq n \leq N}\left\{n^{2-\lambda} a_{n}^{2}\right\} \min _{1 \leq n \leq N}\left\{n^{2-\lambda} b_{n}^{2}\right\}\left[\left(\frac{\pi}{\lambda}\right)^{2}\left(\sum_{n=1}^{N} \frac{1}{n}\right)^{2}-\left(\sum_{n=1}^{N} \sum_{m=1}^{N} \frac{1}{(m n)^{1-\lambda / 2}\left(m^{\lambda}+n^{\lambda}\right)}\right)^{2}\right] .
\end{aligned}
$$

Proof. Let $c_{n}=n^{1-\frac{\lambda}{2}} a_{n}, d_{n}=n^{1-\frac{\lambda}{2}} b_{n}$, then inequality (9) is translated into

$$
\left(\frac{\pi}{\lambda}\right)^{2} \sum_{n=1}^{N} \frac{c_{n}^{2}}{n} \sum_{n=1}^{N} \frac{d_{n}^{2}}{n}-\left(\sum_{n=1}^{N} \sum_{m=1}^{N} \frac{c_{m} d_{n}}{(m n)^{1-\lambda / 2}\left(m^{\lambda}+n^{\lambda}\right)}\right)^{2}
$$




$$
\geq \min _{1 \leq n \leq N}\left\{c_{n}^{2}\right\} \min _{1 \leq n \leq N}\left\{d_{n}^{2}\right\}\left[\left(\frac{\pi}{\lambda}\right)^{2}\left(\sum_{n=1}^{N} \frac{1}{n}\right)^{2}-\left(\sum_{n=1}^{N} \sum_{m=1}^{N} \frac{1}{(m n)^{1-\lambda / 2}\left(m^{\lambda}+n^{\lambda}\right)}\right)^{2}\right] .
$$

By using Cauchy inequality, we have

$$
\begin{aligned}
& \left(\frac{\pi}{\lambda}\right)^{2} \sum_{n=1}^{N} \frac{c_{n}^{2}}{n} \sum_{n=1}^{N} \frac{d_{n}^{2}}{n}-\left(\sum_{n=1}^{N} \sum_{m=1}^{N} \frac{c_{m} d_{n}}{(m n)^{1-\lambda / 2}\left(m^{\lambda}+n^{\lambda}\right)}\right)^{2} \\
& \geq\left(\frac{\pi}{\lambda}\right)^{2} \sum_{n=1}^{N} \frac{c_{n}^{2}}{n} \sum_{n=1}^{N} \frac{d_{n}^{2}}{n}-\sum_{n=1}^{N} \sum_{m=1}^{N} \frac{c_{m}^{2}}{(m n)^{1-\lambda / 2}\left(m^{\lambda}+n^{\lambda}\right)} \sum_{n=1}^{N} \sum_{m=1}^{N} \frac{d_{n}^{2}}{(m n)^{1-\lambda / 2}\left(m^{\lambda}+n^{\lambda}\right)} .
\end{aligned}
$$

Let $f\left(c_{1}, c_{2}, \mathrm{~L}, c_{n}, d_{1}, d_{2}, \mathrm{~L}, d_{n}\right)$

$$
=\left(\frac{\pi}{\lambda}\right)^{2} \sum_{n=1}^{N} \frac{c_{n}^{2}}{n} \sum_{n=1}^{N} \frac{d_{n}^{2}}{n}-\sum_{n=1}^{N} \sum_{m=1}^{N} \frac{c_{m}^{2}}{(m n)^{1-\lambda / 2}\left(m^{\lambda}+n^{\lambda}\right)} \sum_{n=1}^{N} \sum_{m=1}^{N} \frac{d_{n}^{2}}{(m n)^{1-\lambda / 2}\left(m^{\lambda}+n^{\lambda}\right)},
$$

to compute the partial derivatives of a function $f\left(c_{1}, c_{2}, \mathrm{~L}, c_{n}, d_{1}, d_{2}, \mathrm{~L}, d_{n}\right)$ with respect to $c_{i} \cdot d_{i}$, and using lemma 2, we have

$$
\begin{aligned}
& \frac{\partial f}{\partial c_{l}}=\left(\frac{\pi}{\lambda}\right)^{2} \frac{2 c_{i}}{i} \sum_{n=1}^{N} \frac{d_{n}^{2}}{n}-\sum_{n=1}^{N} \frac{2 c_{i}}{i^{1-\lambda / 2} n^{1-\lambda / 2}\left(i^{\lambda}+n^{\lambda}\right)} \sum_{n=1}^{N} \frac{d_{n}^{2}}{n^{1-\lambda / 2}}\left(\sum_{m=1}^{N} \frac{1}{m^{1-\lambda / 2}\left(m^{\lambda}+n^{\lambda}\right)}\right) \\
& >\left(\frac{\pi}{\lambda}\right)^{2} \frac{2 c_{i}}{i} \sum_{n=1}^{N} \frac{d_{n}^{2}}{n}-\frac{2 c_{i}}{i^{1-\lambda / 2}} i^{-\lambda / 2} \frac{\pi}{\lambda} \sum_{n=1}^{N} \frac{d_{n}^{2}}{n^{1-\lambda / 2}}\left(\frac{\pi}{\lambda} n^{-\lambda / 2}\right)=0 .
\end{aligned}
$$

thus $f\left(c_{1}, c_{2}, \mathrm{~L}, c_{n}, d_{1}, d_{2}, \mathrm{~L}, d_{n}\right)$ is monotone increasing for $c_{i}$. In a similar way we can provde that $\frac{\partial f}{\partial d_{i}}>0$, and this implies $f\left(c_{1}, c_{2}, \mathrm{~L}, c_{n}, d_{1}, d_{2}, \mathrm{~L}, d_{n}\right)$ is monotone increasing for $d_{i}$. We obtain $f\left(c_{1}, c_{2}, \mathrm{~L}, c_{n}, d_{1}, d_{2}, \mathrm{~L}, d_{n}\right)$

$\geq f\left(\min _{1 \leq n \leq N}\left\{c_{n}\right\}, \min _{1 \leq n \leq N}\left\{c_{n}\right\}, \mathrm{L}, \min _{1 \leq n \leq N}\left\{c_{n}\right\}, \min _{1 \leq n \leq N}\left\{d_{n}\right\}, \min _{1 \leq n \leq N}\left\{d_{n}\right\}, \mathrm{L}, \min _{1 \leq n \leq N}\left\{d_{n}\right\}\right)$.

In view of (11), (10) holds. The theorem is proved.

Theorem 2 If $a_{n} \geq 0, b_{n} \geq 0, n=0,1,2, \mathrm{~L}, N .0<\lambda \leq 1$, then

$$
\begin{aligned}
& \left(\frac{\pi}{\lambda}\right)^{2} \sum_{n=0}^{N}(n+1 / 2)^{1-\lambda} a_{n}^{2} \sum_{n=0}^{N}(n+1 / 2)^{1-\lambda} b_{n}^{2}-\left(\sum_{n=0}^{N} \sum_{m=0}^{N} \frac{a_{m} b_{n}}{(m+1 / 2)^{\lambda}+(n+1 / 2)^{\lambda}}\right)^{2} \\
& \geq \min _{0 \leq n \leq N}\left\{(n+1 / 2)^{2-\lambda} a_{n}^{2}\right\} \min _{0 \leq n \leq N}\left\{(n+1 / 2)^{2-\lambda} b_{n}^{2}\right\} \\
& \times\left[\left(\frac{\pi}{\lambda}\right)^{2}\left(\sum_{n=0}^{N} \frac{1}{n+1 / 2}\right)^{2}-\left(\sum_{n=0}^{N} \sum_{m=0}^{N} \frac{1}{(n+1 / 2)^{1-\lambda / 2}(m+1 / 2)^{1-\lambda / 2}\left((m+1 / 2)^{\lambda}+(n+1 / 2)^{\lambda}\right)}\right)^{2}\right] .
\end{aligned}
$$

Proof. Let $c_{n}=(n+1 / 2)^{1-\frac{\lambda}{2}} a_{n}, d_{n}=(n+1 / 2)^{1-\frac{\lambda}{2}} b_{n}$, then inequality (12) is translated into

$$
\left(\frac{\pi}{\lambda}\right)^{2} \sum_{n=0}^{N} \frac{c_{n}^{2}}{n+1 / 2} \sum_{n=0}^{N} \frac{d_{n}^{2}}{n+1 / 2}-\left(\sum_{n=0}^{N} \sum_{m=0}^{N} \frac{c_{m} d_{n}}{(n+1 / 2)^{1-\lambda / 2}(m+1 / 2)^{1-\lambda / 2}\left((m+1 / 2)^{\lambda}+(n+1 / 2)^{\lambda}\right)}\right)^{2}
$$




$$
\geq \min _{0 \leq n \leq N}\left\{c_{n}^{2}\right\} \min _{0 \leq n \leq N}\left\{d_{n}^{2}\right\}\left[B^{2}\left(\frac{\lambda}{2}, \frac{\lambda}{2}\right)\left(\frac{\pi}{\lambda}\right)^{2}-\left(\sum_{n=0}^{N} \sum_{m=0}^{N} \frac{1}{(n+1 / 2)^{1-\lambda / 2}(m+1 / 2)^{1-\lambda / 2}\left((m+1 / 2)^{\lambda}+(n+1 / 2)^{\lambda}\right)}\right)^{2}\right] .
$$

By using Cauchy inequality, we have

$$
\begin{aligned}
& \left(\frac{\pi}{\lambda}\right)^{2} \sum_{n=0}^{N} \frac{c_{n}^{2}}{n+1 / 2} \sum_{n=0}^{N} \frac{d_{n}^{2}}{n+1 / 2}-\left(\sum_{n=0}^{N} \sum_{m=0}^{N} \frac{c_{m} d_{n}}{(n+1 / 2)^{1-\lambda / 2}(m+1 / 2)^{1-\lambda / 2}\left((m+1 / 2)^{\lambda}+(n+1 / 2)^{\lambda}\right)}\right)^{2} \\
& >\left(\frac{\pi}{\lambda}\right)^{2} \sum_{n=0}^{N} \frac{c_{n}^{2}}{n+1 / 2} \sum_{n=0}^{N} \frac{d_{n}^{2}}{n+1 / 2} \\
& -\sum_{n=1}^{N} \sum_{m=1}^{N} \frac{c_{m}^{2}}{(n+1 / 2)^{1-\lambda / 2}(m+1 / 2)^{1-\lambda / 2}\left((m+1 / 2)^{\lambda}+(n+1 / 2)^{\lambda}\right)^{n}} \sum_{n=1}^{N} \sum_{m=1}^{N} \frac{d_{n}^{2}}{(n+1 / 2)^{1-\lambda / 2}(m+1 / 2)^{1-\lambda / 2}\left((m+1 / 2)^{\lambda}+(n+1 / 2)^{\lambda}\right)}
\end{aligned}
$$

To define a function $f\left(c_{1}, c_{2}, \mathrm{~L}, c_{n}, d_{1}, d_{2}, \mathrm{~L}, d_{n}\right)$ by the right hand side of (14), computting the partial derivatives of a function $f\left(c_{1}, c_{2}, \mathrm{~L}, c_{n}, d_{1}, d_{2}, \mathrm{~L}, d_{n}\right)$ with respect to $c_{i} \cdot d_{i}$, and using lemma 3 , we have

$$
\begin{aligned}
& \frac{\partial f}{\partial c_{l}}=\left(\frac{\pi}{\lambda}\right)^{2} \frac{2 c_{i}}{i+1 / 2} \sum_{n=0}^{N} \frac{d_{n}^{2}}{n+1 / 2} \\
& -\sum_{n=0}^{N} \frac{2 c_{i}}{(i+1 / 2)^{1-\lambda / 2}(n+1 / 2)^{1-\lambda / 2}\left((i+1 / 2)^{\lambda}+(n+1 / 2)^{\lambda}\right)^{n=1}} \sum_{n}^{N} \frac{d_{n}^{2}}{(n+1 / 2)^{1-\lambda / 2}}\left(\sum_{m=0}^{N} \frac{1}{(m+1 / 2)^{1-\lambda / 2}\left((m+1 / 2)^{\lambda}+(n+1 / 2)^{\lambda}\right)}\right) \\
& >\left(\frac{\pi}{\lambda}\right)^{2} \frac{2 c_{i}}{i+1 / 2} \sum_{n=0}^{N} \frac{d_{n}^{2}}{n+1 / 2}-\frac{2 c_{i}}{(i+1 / 2)^{1-\lambda / 2}}(i+1 / 2)^{-\lambda / 2} \frac{\pi}{\lambda} \sum_{n=0}^{N} \frac{d_{n}^{2}}{(n+1 / 2)^{1-\lambda / 2}}\left(\frac{\pi}{\lambda}(n+1 / 2)^{-\lambda / 2}\right)=0,
\end{aligned}
$$

and $\frac{\partial f}{\partial d_{l}}>0$, thus $f\left(c_{1}, c_{2}, \mathrm{~L}, c_{n}, d_{1}, d_{2}, \mathrm{~L}, d_{n}\right)$ is monotone increasing for $c_{i}, d_{i}$. We get $f\left(c_{1}, c_{2}, \mathrm{~L}, c_{n}, d_{1}, d_{2}, \mathrm{~L}, d_{n}\right) \geq f\left(\min _{1 \leq n \leq N}\left\{c_{n}\right\}, \mathrm{L}, \min _{1 \leq n \leq N}\left\{c_{n}\right\}, \min _{1 \leq n \leq N}\left\{d_{n}\right\}, \mathrm{L}, \min _{1 \leq n \leq N}\left\{d_{n}\right\}\right)$. In view of (14), (13) holds. The theorem is proved.

\section{References}

[1] G. H. Hardy, J. E. Littlewood and G. Pólya, Inequalities, Gambridge University Press, Gambridge, 1952.

[2] X. Zhang, Y. Chu, New discussion to analytic inequalities, Harbin institute of technology press,2009.

[3] M. Gao, B. Yang, On the extended Hilbert's inequality, Proceeding of the American Mathematical Society 126 (1998), no.3, 751-759

[4] J. Kuang, L. Debnath, On new generalizations of Hilbert's inequality and their applications, Journal of Mathematical Analysis and Applications 245 (2000), no.1, 248-265.

[5] B. Yang, On a strengthened version of the more accurate Hardy-Hilbert's inequality, Acta Mathematica, Sinica (China) 42 (1999), no.6, 1103-1110. 